\title{
Autopercepção de saúde de idosos usuários de um serviço de oncologia
}

Health self-perception among older adults users of an oncology service

Autopercepción de salud de ancianos atendidos en un servicio de oncología

Raquel Heling Reis ${ }^{1}$, Eda Schwartz ${ }^{2}$, Lílian Moura de Lima ${ }^{3}$, Celmira Lange ${ }^{4}$, Aline Machado Feijó ${ }^{5}$, Giani Cunha Duarte ${ }^{6}$

${ }^{1}$ Enfermeira. Enfermeira do Hospital Universitário São Francisco de Paula da Universidade Católica de Pelotas. Pelotas, RS, Brasil. E-mail:

raquelreling@hotmail.com.

${ }^{2}$ Enfermeira, Doutora em Enfermagem. Professora Associada da Universidade Federal de Pelotas (UFPel). Pelotas, RS, Brasil. E-mail:

eschwartz@terra.com.br.

${ }^{3}$ Enfermeira, Mestre em Enfermagem. Discente do Programa de Pós-Graduação em Enfermagem, nível Doutorado, da UFPel. Pelotas, RS, Brasil. E-mail: lima.lilian@gmail.com.

${ }^{4}$ Enfermeira, Doutora em Enfermagem. Professora Adjunta da UFPel. Pelotas, RS, Brasil. E-mail: celmira_lange@terra.com.br.

${ }^{5}$ Enfermeira, Mestre em Enfermagem. Discente do Programa de Pós-Graduação em Enfermagem, nível Doutorado, da UFPel. Pelotas, RS, Brasil. E-mail:

aline_feijo@yahoo.com.br.

${ }^{6}$ Enfermeira. Discente do Programa de Pós-Graduação em Enfermagem, nível Mestrado, da UFPel. Pelotas, RS, Brasil. E-mail: giani_cd@hotmail.com.

\section{RESUMO}

Objetivou-se descrever a autopercepção de saúde dos idosos em tratamento quimioterápico em um serviço de oncologia, segundo variáveis sociodemográficas, morbidades e apoio social. Estudo quantitativo, descritivo realizado com aplicação de questionário pré-codificado, com perguntas fechadas, tendo como respondentes 108 idosos em tratamento quimioterápico. Os dados foram organizados em um banco, e a análise feita por distribuição de frequência. Dentre os resultados sobre a saúde autorreferida, destaca-se a melhor avaliação de saúde nos indivíduos do sexo masculino que vivem com companheiro e realizam algum tipo de trabalho. Já os piores resultados obtidos foram na faixa etária de 60 a 74 anos, habitantes da zona rural, com renda de um a dois salários mínimos e apresentam hipertensão arterial sistêmica. As descobertas apontam possibilidades de intervenção para os profissionais de saúde, podendo fomentar a melhora da saúde percebida, dentre as quais estão o estímulo ao apoio familiar e a manutenção de atividades laborais.

Descritores: Doença Crônica; Saúde do Idoso; Oncologia, Enfermagem Geriátrica.

\section{ABSTRACT}

We aimed at describing health self-perception of older adults receiving chemotherapy in an oncology service, according to sociodemographic, morbidity and social support variables. A quantitative descriptive study conducted by applying pre-coded questionnaires with close-ended questions, which were given to 108 older adults receiving chemotherapy. The data were organized in a bank and analyzed through frequency distribution. Results displayed that male participants who lived with a partner and had some type of occupation had higher self-reported health levels. The poorest results were obtained in the 60-74 age group, among residents of rural areas, with one to two minimum monthly wages and systemic arterial hypertension. The results suggest intervention possibilities for health professionals, which can lead to improving self-perceived health status, such as stimulating family support and maintaining occupational activities.

Descriptors: Chronic Disease; Health of the Elderl; Medical Oncology; Geriatric Nursing.

\section{RESUMEN}

Se objetivó describir la autopercepción de salud de ancianos en tratamiento quimioterápico en un servicio de oncología según variables sociodemográficas, morbilidades y apoyo social. Estudio cuantitativo, descriptivo, con aplicación de cuestionario preconcebido con preguntas cerradas, al que respondieron 108 ancianos en tratamiento quimioterápico. Se organizó banco de datos, analizado mediante distribución de frecuencia. Entre los resultados autorreferidos de salud, destaca la mejor evaluación de salud en individuos de sexo masculino, viviendo en pareja y realizando algún tipo de trabajo. Los peores resultados obtenidos correspondieron a la faja etaria de 60 a 74 años, residentes en zona rural, ingresos de uno a dos salarios mínimos, presentando hipertensión arterial sistémica. Los hallazgos expresan posibilidades de intervención para los profesionales de salud, pudiendo fomentar la mejora de salud percibida, entre las que está el estímulo del apoyo familiar y el mantenimiento de actividades laborales.

Descriptores: Enfermedad Crónica; Salud del Anciano; Oncología Médica; Enfermería Geriátrica. 


\section{INTRODUÇÃO}

Consideram-se idosos os indivíduos com 60 anos ou mais $^{(1)}$. Esta população, no Brasil, passou de três milhões em 1960, para sete milhões em 1975 e 17 milhões em 2006, representando um aumento de $600 \%$ em menos de 50 anos. A cada ano aproximadamente 650.000 idosos são incorporados a este grupo, a maior parte deles com doenças crônicas não transmissíveis $(\mathrm{DCNT})^{(2)}$.

Dentre as DCNT, o câncer constitui-se na segunda principal causa de morte no mundo, representando $13 \%$ do total de óbitos anuais ${ }^{(3)}$. No Brasil, a estimativa do Instituto Nacional de Câncer (INCA) para o ano de 2014 é de 394.450 casos novos da doença ${ }^{(4)}$. Em pesquisa que analisou a mortalidade entre os idosos brasileiros, no ano de 2007, os autores identificaram que as neoplasias $(17,1 \%)$ estão em segundo lugar, perdendo apenas para as doenças do aparelho circulatório (42,2\%), constituindo-se um importante problema de saúde para esta faixa etária( ${ }^{(5)}$.

Diante das proporções elevadas de adoecimento por câncer na população idosa, é imprescindível conhecer as influências da enfermidade sobre as condições de saúde destes sujeitos. Para tanto, pode-se considerar a avaliação da autopercepção de saúde como um importante indicador ${ }^{(6)}$. A autopercepção de saúde é o produto de um amplo espectro de fatores determinantes, que incluem características sociodemográficas e aspectos relacionados às condições de vida e saúde, podendo refletir o estado do corpo e da mente humana, além do comportamento em saúde e o bem-estar social ${ }^{(6-8)}$. Reconhece-se ainda a relação entre a autopercepção de saúde e o autocuidado, a mortalidade, a morbidade, e o uso de serviços de saúde ${ }^{(9-11)}$.

Estudos realizados com idosos brasileiros e coreanos $^{(12-13)}$ apontam que a autossatisfação com a saúde esteve relacionada a estar satisfeito com as atividades da vida diária, com a vida em geral, com a autoestima, com o lazer e, ainda, a não possuir DCNT. Em estudo ${ }^{(9)}$ realizado no estado de Minas Gerais com 98 idosos, averiguou-se a relação entre a auto-percepção de saúde e o estado cognitivo. Observou-se que dentre aqueles idosos sem demência, mas com eventos cerebrovasculares anteriores, houve uma pior autoavaliação de saúde, confirmando que os sujeitos com DCNT tendem a avaliar sua saúde de forma negativa.

Diante do exposto, observou-se a publicação atualizada relacionada a auto-avaliação de saúde, bemestar e satisfação com a vida entre idosos ${ }^{(6-7,9,13-16)}$, entretanto nota-se carência na literatura de estudos que incluam a autopercepção de saúde em idosos com câncer. Dessa forma, o presente estudo pretende oferecer subsídios aos profissionais envolvidos na atenção aos idosos acometidos por câncer, favorecendo a qualificação para o cuidado integral, com intervenções voltadas para a modificação das variáveis que podem representar uma pior auto-avaliação de saúde. Para tanto se objetivou descrever a autopercepção de saúde dos idosos em tratamento quimioterápico em um serviço de oncologia, segundo variáveis sociodemográficas, morbidades e apoio social.

\section{METODOLOGIA}

Este estudo trata de uma investigação quantitativa descritiva, realizada em um Centro Regional de Oncologia da região sul do Brasil. Esse centro recebe pessoas de ambos os sexos que estão em tratamento para algum tipo de câncer, e realiza o atendimento médio de 20 pacientes por dia, com 250 novos usuários a cada ano.

A amostra foi composta por 108 idosos em tratamento quimioterápico no referido serviço de saúde, no período compreendido entre março e junho de 2010, caracterizando-se em uma amostra de conveniência. Os critérios de inclusão foram: idade maior ou igual a 60 anos, estar ciente do diagnóstico de câncer e estar em tratamento no Centro Regional de Oncologia. Aqueles clientes que se enquadraram nos critérios de inclusão foram convidados a participar da pesquisa.

Os dados foram coletados aplicando-se um questionário pré-codificado, contendo 94 questões, por acadêmicos que receberam uma capacitação prévia do pesquisador responsável. Do total de questões foram utilizadas 21 variáveis para a composição deste artigo, sendo: sexo (feminino/masculino), raça (branca/preta/parda/amarela/indígena), estado conjugal (sim, vive com companheiro/não vive com companheiro/não, mas já viveu com companheiro), procedência (rural/ urbana), escolaridade (em anos completos de estudos), trabalho remunerado (sim/não), trabalho não remunerado ( $\operatorname{sim} /$ não), renda familiar (em salários mínimos), religião (católica/protestante/espírita/umbanda/evangélica/pente costal), filhos (em números), cardiopatia (sim/não), hipertensão arterial sistêmica (sim/não), diabetes mellitus (sim/não), situação geral de saúde (péssima, má, regular, boa e ótima), frequenta grupo de apoio (sim/não), possui alguém que possa the cuidar (sim/não).

As informações foram digitadas em um banco de 
dados construído no software Epi info 6.04, com dupla entrada de dados e pareamento dos bancos para correção de inconsistências. Após conferência, os dados foram analisados neste mesmo programa, utilizando-se estatística básica com distribuição de frequência das variáveis, estratificando-as pela saúde autorreferida.

A pesquisa respeitou os princípios éticos que norteiam a pesquisa com seres humanos e foi submetida à apreciação do Comitê de Ética em Pesquisa recebendo parecer favorável sob o no 028/08. Os participantes do estudo foram informados sobre o direito de desistir da participação a qualquer momento e assinaram o Termo de Consentimento Livre e Esclarecido.

\section{RESULTADOS}

$\mathrm{Na}$ Tabela 1 verifica-se a distribuição das características sociodemográficas da amostra de idosos estratificada pela saúde autorreferida. Observa-se que predominou entre os idosos a referência à saúde como regular em ambos os sexos. Quanto à faixa etária notase que entre 60 a 74 anos $54,2 \%(n=45)$ referiram seu estado de saúde como regular, já na faixa etária de 75 anos ou mais houve distribuição idêntica dos informantes, que consideraram sua saúde como regular e boa/ótima com 48,0\% ( $n=12)$.

Tabela 1: Distribuição das variáveis sociodemográficas estratificada pela saúde autorreferida em idosos em tratamento quimioterápico $(n=108)$. Região Sul do Brasil, 2011.

\begin{tabular}{|c|c|c|c|c|c|c|c|c|}
\hline \multirow{3}{*}{ Características } & \multicolumn{8}{|c|}{ Saúde autorreferida } \\
\hline & \multicolumn{2}{|c|}{ Total } & \multicolumn{2}{|c|}{ Péssima/má } & \multicolumn{2}{|c|}{ Regular } & \multicolumn{2}{|c|}{ Boa/ótima } \\
\hline & $\mathbf{n}$ & $(\%)$ & $\mathbf{n}$ & $(\%)$ & $\mathbf{n}$ & $(\%)$ & $\mathbf{n}$ & $(\%)$ \\
\hline \multicolumn{9}{|l|}{ Sexo } \\
\hline Masculino & 62 & 57,4 & 3 & 4,8 & 30 & 48,4 & 29 & 46,8 \\
\hline Feminino & 46 & 42,6 & 1 & 2,2 & 27 & 58,7 & 18 & 39,1 \\
\hline \multicolumn{9}{|l|}{ Faixa etária } \\
\hline 60 a 74 anos & 83 & 76,9 & 3 & 3,6 & 45 & 54,2 & 35 & 42,2 \\
\hline 75 anos ou mais & 25 & 23,1 & 1 & 4 & 12 & 48 & 12 & 48 \\
\hline \multicolumn{9}{|l|}{ Procedência } \\
\hline Rural & 52 & 48,1 & 1 & 1,9 & 29 & 55,8 & 22 & 42,3 \\
\hline Urbana & 56 & 51,9 & 3 & 5,4 & 28 & 50 & 25 & 44,6 \\
\hline \multicolumn{9}{|l|}{ Vive com companheiro } \\
\hline Não & 38 & 35.2 & 1 & 2,6 & 24 & 63,2 & 13 & 34,2 \\
\hline Sim & 70 & 64.8 & 3 & 4,3 & 33 & 47,1 & 34 & 48,6 \\
\hline \multicolumn{9}{|l|}{ Escolaridade } \\
\hline Sem escolaridade & 16 & 15 & 1 & 6,3 & 8 & 50 & 7 & 43,7 \\
\hline 1 a 4 anos & 52 & 48,6 & 2 & 3,9 & 26 & 50 & 24 & 46,1 \\
\hline 5 a 8 anos & 30 & 28 & 1 & 3,3 & 17 & 56,7 & 12 & 40 \\
\hline 9 a 12 anos & 9 & 8,4 & 0 & 0 & 5 & 55,6 & 4 & 44,4 \\
\hline \multicolumn{9}{|l|}{ Trabalho remunerado } \\
\hline Não & 94 & 87 & 4 & 4,3 & 52 & 55,3 & 38 & 40,4 \\
\hline Sim & 14 & 13 & 0 & 0 & 5 & 35,7 & 9 & 64,3 \\
\hline \multicolumn{9}{|l|}{ Trabalho não-remunerado } \\
\hline Não & 85 & 78,7 & 4 & 4,7 & 48 & 56,5 & 33 & 38,8 \\
\hline Sim & 23 & 21,3 & 0 & 0 & 9 & 39,1 & 14 & 60,9 \\
\hline \multicolumn{9}{|l|}{ Fonte de renda } \\
\hline Aposentadoria/benefício & 94 & 87 & 4 & 3,7 & 52 & 55,3 & 38 & 40,4 \\
\hline Renda familiar & 11 & 10,2 & 0 & 0 & 5 & 45,5 & 6 & 54,6 \\
\hline Emprego & 3 & 2,8 & 0 & 0 & 0 & 0 & 3 & 100 \\
\hline \multicolumn{9}{|c|}{ Renda familiar (em salários mínimos)* } \\
\hline 1 a 2 & 60 & 60,6 & 4 & 6,7 & 32 & 53,3 & 24 & 40 \\
\hline 3 a 4 & 32 & 32,3 & 0 & 0 & 18 & 56,3 & 14 & 43,8 \\
\hline 5 a mais & 7 & 7,1 & 0 & 0 & 4 & 57,1 & 3 & 42,9 \\
\hline \multicolumn{9}{|l|}{ Religião } \\
\hline Possui & 95 & 12 & 3 & 3,2 & 49 & 51,5 & 43 & 45,3 \\
\hline Não possui & 13 & 78 & 1 & 7,7 & 8 & 61,5 & 4 & 30,8 \\
\hline
\end{tabular}

Quando se distribuiu a saúde autorreferida pelo local de procedência dos idosos, observou-se que tanto os da zona rural como os da urbana, consideraram predominantemente sua saúde como regular.
Os idosos que relataram viver com companheiro apontaram sua saúde como boa/ótima em 48,6\% $(n=34)$ dos casos, enquanto que aqueles que vivem sozinhos consideraram seu estado de saúde como regular em 


$$
63,2 \%(n=24) \text {. }
$$

Quando se distribuiu a saúde autorreferida pelo grau de escolaridade, observou-se que os idosos referiram-na, predominantemente como regular, independente dos anos de estudo.

Em relação ao trabalho, nenhum dos idosos que disseram desenvolver atividades remuneradas ou não remuneradas relatou sua saúde como péssima/má, sendo que houve predomínio em apontar sua saúde como boa/ótima. A fonte de renda foi outra variável analisada de acordo com a saúde autorreferida, a principal fonte de renda para os idosos entrevistados foi a aposentadoria, sendo que 55,3\% $(n=52)$ destes consideraram seu estado de saúde como regular. Daqueles que apresentaram a renda familiar como principal fonte de subsistência, os resultados foram de $54,6 \% \quad(n=6)$ saúde autorreferida como boa/ótima, levando em consideração que nenhum referiu a saúde de forma negativa.

Distribuindo a saúde autorreferida pela variável renda familiar, os idosos que possuem de um a dois salários mínimos mencionaram a própria saúde como regular, sendo o único grupo a apontá-la como péssima/má.

Quanto a distribuição da autopercepção de saúde pela variável religião, observou-se que tanto os que possuem como aqueles que não possuem crença religiosa consideraram predominantemente sua saúde autorreferida como regular.

Dentre as morbidades referidas pelos participantes, incluídas na Tabela 2, observa-se a predominância da hipertensão arterial sistêmica nos idosos entrevistados, seguida pelas doenças do coração e pelo diabetes mellitus.

Tabela 2: Distribuição das variáveis de doenças pré-existentes estratificada pela saúde autorreferida em idosos em tratamento quimioterápico $(n=108)$. Região Sul do Brasil, 2011.

\begin{tabular}{|c|c|c|c|c|c|c|c|c|}
\hline \multirow{3}{*}{ Características } & \multicolumn{8}{|c|}{ Saúde autorreferida } \\
\hline & \multicolumn{2}{|c|}{ Total } & \multicolumn{2}{|c|}{ Péssima/má } & \multicolumn{2}{|c|}{ Regular } & \multicolumn{2}{|c|}{ Boa/ótima } \\
\hline & $\mathbf{n}$ & $\%$ & $\mathbf{n}$ & $\%$ & $\mathbf{n}$ & $\%$ & $\mathbf{n}$ & $\%$ \\
\hline \multicolumn{9}{|l|}{ Doenças do coração } \\
\hline Não & 86 & 80,4 & 4 & 4,7 & 45 & 52,3 & 37 & 43 \\
\hline $\operatorname{Sim}$ & 21 & 19,6 & 0 & 0 & 11 & 52,4 & 10 & 47,6 \\
\hline \multicolumn{9}{|l|}{ Diabetes Mellitus } \\
\hline Não & 89 & 82,4 & 4 & 4,5 & 44 & 49,4 & 41 & 46,1 \\
\hline Sim & 19 & 17,6 & 0 & 0 & 13 & 68,4 & 6 & 31,6 \\
\hline \multicolumn{9}{|l|}{ Hipertensão arterial sistêmica } \\
\hline Não & 62 & 57,4 & 3 & 4,8 & 33 & 53,2 & 26 & 42 \\
\hline Sim & 46 & 42,6 & 1 & 2,2 & 24 & 52,2 & 21 & 45,6 \\
\hline
\end{tabular}

Ao distribuir as doenças pré-existentes pela saúde autorreferida, pode-se observar que nenhum dos idosos com câncer que possui doenças do coração e diabetes mellitus referiu sua saúde atual como péssima/má. Já entre os idosos hipertensos apenas $2,2 \% \quad(n=1)$ autorreferiram sua saúde como péssima.

Tabela 3: Distribuição das variáveis redes de apoio por saúde autorreferida em idosos em tratamento quimioterápico $(n=108)$. Região Sul do Brasil, 2011.

\begin{tabular}{|c|c|c|c|c|c|c|c|c|}
\hline \multirow{3}{*}{ Características } & \multicolumn{8}{|c|}{ Saúde autorreferida } \\
\hline & \multicolumn{2}{|c|}{ Total } & \multicolumn{2}{|c|}{ Péssima/má } & \multicolumn{2}{|c|}{ Regular } & \multicolumn{2}{|c|}{ Boa/ótima } \\
\hline & $\mathbf{n}$ & $\%$ & $\mathbf{N}$ & $\%$ & $\mathbf{n}$ & $\%$ & $\mathbf{n}$ & $\%$ \\
\hline \multicolumn{9}{|c|}{ Frequenta grupo/associação de apoio } \\
\hline Não & 102 & 94,4 & 4 & 3,9 & 52 & 51 & 46 & 45,1 \\
\hline Sim & 6 & 5,6 & 0 & 0 & 5 & 83,3 & 1 & 16,7 \\
\hline \multicolumn{9}{|l|}{ Possui cuidador } \\
\hline Não & 4 & 3,7 & 0 & 0 & 3 & 75 & 1 & 25 \\
\hline Sim & 104 & 96,3 & 4 & 3,9 & 54 & 51,9 & 46 & 44,2 \\
\hline
\end{tabular}

Na Tabela 3, verifica-se que 94,4\% $(n=102)$ dos idosos relataram não frequentar nenhum grupo de apoio e 96,3\% ( $n=104)$ disseram possuir alguém que cuida ou poderia cuidar caso fosse necessário. Destaca-se, ainda, que 0 estado de saúde autorreferido foi predominantemente o regular para as duas variáveis.

\section{DISCUSSÃo}

O atendimento ao idoso com câncer é um desafio para as equipes de saúde, visto que, associados à doença existem os fatores fisiológicos limitantes, próprios desta faixa etária, que, muitas vezes, causam 
dependência e levam a auto-avaliação negativa da saúde ${ }^{(12)}$. Sendo assim, conhecer o perfil do idoso e como ele auto-avalia o seu estado de saúde favorecem a assistência integral, estimulando o autocuidado, a melhora do estado de saúde percebido e instiga-os a redescobrir possibilidades e desfrutar de melhor qualidade de vida ${ }^{(15-17)}$.

Ao analisar os resultados deste estudo, observou-se que estratificando a saúde autorreferida pelo sexo, os homens concentraram-se proporcionalmente entre as categorias de saúde regular e ótima. O mesmo resultado foi observado em estudo com idosos realizado na cidade de São Paulo(11), no qual se encontrou que o sexo masculino tinha um menor risco de auto-avaliar a saúde como ruim quando comparado ao feminino, e que entre os indivíduos de 60 anos ou mais a chance de autoavaliar a saúde como ruim foi $16 \%$ maior para o sexo feminino do que para o masculino.

Estudo multicêntrico realizado em sete cidades brasileiras $^{(14)}$ identificou que as mulheres experimentam um maior número de doenças crônicas, muitas vezes incapacitantes. Este fato aliado a maior expectativa de vida das mulheres, proporciona uma convivência prolongada com as DCNT, favorecendo a avaliação negativa de sua saúde. Assim, a auto-avaliação negativa da saúde feita pelas mulheres com câncer guarda relação com a qualidade de vida, enquanto que os homens quando avaliam sua saúde de forma negativa apresentam risco maior de serem acometidos por um evento fatal.

Uma investigação sobre a saúde autorreferida realizada no Ceará(17) identificou relação entre a idade e a autopercepção de saúde. Os pesquisadores verificaram que os idosos consideraram sua saúde como regular/ruim na proporção de $42,2 \%(n=92)$ para a faixa etária de 60-69 anos, e de 54,5\% $(n=79)$ para a de 7079 anos. $\mathrm{Na}$ presente pesquisa observou-se que os idosos entrevistados, independentemente da faixa etária, concordaram em apontar sua saúde de regular a boa/ótima.

O meio físico e sociocultural onde o indivíduo está inserido interfere em sua auto-avaliação de saúde, sendo que os idosos nascidos no meio rural apresentaram uma frequência maior de autopercepção negativa de seu estado de saúde, quando comparado com os que nasceram na zona urbana(8). Esta afirmativa vai de encontro ao identificado na presente amostra de idosos.

Outro fator relevante na autopercepção de saúde é o estado conjugal ${ }^{(13-16)}$, sendo os indivíduos casados mais propensos a terem melhor avaliação sobre sua saúde, seguidos pelos solteiros, os viúvos e os divorciados. Isso corrobora com o observado na presente pesquisa, pois os idosos que referiram viver com cônjuge/companheiro, auto-avaliaram sua saúde como ótima, enquanto que entre aqueles que viviam sozinhos predominou o estado regular. Este resultado enfatiza a relevância do convívio familiar para o idoso, principalmente em situação de enfermidade grave como o câncer, pois é na família que o idoso irá apoiar-se para estabelecer uma condição de vida saudável.

A variável escolaridade é um importante fator de proteção para a avaliação negativa da saúde(18), acreditando-se que a chance de o idoso analfabeto autoavaliar sua saúde de forma negativa é maior do que para os idosos alfabetizados(8-16). Tal aspecto é consolidado por resultados de estudo realizado em Botucatu/SP(12), no qual os idosos com maior escolaridade tiveram uma melhor percepção do seu estado de saúde. Entretanto, no presente estudo não se observou diferença na distribuição das proporções de auto-avaliação da saúde por escolaridade.

No Brasil foi realizada a avaliação comparativa da autopercepção de saúde entre idosos nos anos de 1998 e 2008, obtendo-se como resultado que os idosos entrevistados em 2008 avaliaram sua saúde positivamente, independendo dos estratos de renda(6). Enquanto que no presente estudo observa-se que houve presença de auto-avaliação de péssima a má apenas na faixa de renda menor. A presença do diagnóstico de câncer traz consigo muitos gastos com a saúde, embora o tratamento seja fornecido pelo Sistema Único de Saúde, há necessidade de uma alimentação especial e gastos com transporte, que se tornam dispendiosos para aqueles que contam com pouco recurso financeiro, podendo induzi-los a auto-avaliarem sua condição de forma mais negativa.

Sabe-se que a atuação em trabalhos voluntários serve como um mecanismo de promoção da saúde, devido ao prestígio social, a amizade, a alegria, as gratificações pessoais e os múltiplos papéis proporcionados pelo voluntariado, os quais parecem convergir para que o voluntário sinta-se mais saudável ${ }^{(19)}$. Portanto, a autopercepção, segundo essa variável, pode ser considerada um viés, pois possivelmente eles consigam desenvolver 0 trabalho justamente porque têm sua saúde como boa/ótima. Em estudo $^{(19)}$ que comparou grupos de idosos voluntariados e não voluntariados quanto a autopercepção da saúde, obteve-se o resultado de auto-avaliação de saúde como ótimo em $30,5 \%$ para o primeiro grupo, e $6,1 \%$ para o 
segundo.

A religião é uma importante dimensão da qualidade de vida, entretanto, nos entrevistados não houve diferenças na distribuição da autopercepção de saúde, predominando a saúde como regular ${ }^{(20)}$. Considera-se que a religião proporcione um suporte aos doentes com câncer, visto que, a presença da enfermidade, muitas vezes, suscita a possibilidade da finitude.

Investigação realizada com população idosa em cidade do Ceará(18) mostrou que a saúde autorreferida razoável/ruim se mostrou associada positivamente a todas as condições crônicas pesquisadas, e foi observado que quanto maior o número de co-morbidades, maior o risco de auto-avaliação negativa da saúde. Em contrapartida, um estudo ${ }^{(8)}$ relatou que pessoas portadoras de algum tipo de doença crônica também podem referir uma boa saúde, e ainda afirma que o câncer não modificou a autopercepção de saúde dos idosos. Esse fato pode ser explicado, pois os pacientes oncológicos tendem a aprofundar seus conhecimentos sobre a doença no decorrer do tratamento, o que vai modificando sua autopercepção sobre seu estado de saúde ${ }^{(21)}$.

A atenção e o suporte oferecido pelo cuidador fazem o paciente oncológico enfrentar as dificuldades do caminho com segurança e força, ressaltando que os familiares desenvolvem papel importante na vida do doente em todos os aspectos, sejam estes sociais, emocionais ou afetivos ${ }^{(15-22)}$. Além disso, observa-se que os idosos com menor envolvimento familiar e social estão mais propensos a ter insatisfação com a vida de forma geral $^{(14)}$. Na presente pesquisa observa-se concordância com os autores supracitados, visto que, dentre os entrevistados que referiram participar de grupos de apoio não há referência a auto-avaliação do estado de saúde como péssima/má, assim como, houve predomínio de entrevistados que referiram possuir

\section{REFERÊNCIAS}

1. Ministério do desenvolvimento social e combate à fome. Política Nacional do Idoso. Brasília, 2010. [citado 2014 mar 14]. Disponível em:

http://www.mds.gov.br/assistenciasocial/secretaria-nacional-deassistencia-social-snas/cadernos/politica-nacional-doidoso/Politica\%20Nacional\%20do\%20Idoso.pdf/view?searchterm =sa $\%$ C3\%BAde $\% 20$ do $\% 20$ idoso.

2. Fêde A, Miranda V, Pecoroni P, Fraile N, Santos M, Gonzaga S, Luiz O, Riechelmann R, Giglio A. A importância das neoplasias na população idosa brasileira de 2000 a 2005 . Einstein.

2009;7(2):14-6.

3. Goulart FAA. Organização Pan-americana de saúde. Doenças crônicas não transmissíveis: estratégias de controle e desafios para os sistemas de saúde. Brasília, 2011. [citado 2014 mar 16]. Disponível em: http://apsredes.org/site2012/wp- cuidador e consideram seu estado de saúde como regular.

\section{CONSIDERAÇÕES FINAIS}

A elaboração da presente pesquisa, permitiu conhecer como os idosos em tratamento quimioterápico no serviço em estudo, autorreferem sua saúde segundo as variáveis sociodemográficas, morbidades e suporte social. Dentre os resultados encontrados sobre a saúde autorreferida, pode-se observar que o sexo masculino, viver com companheiro e realizar algum tipo de trabalho, seja remunerado ou não, apresentaram melhores resultados na auto-avaliação da própria saúde, já a faixa etária de 60 a 74 anos, viver na zona rural, possuir renda de um a dois salários mínimos e ser portador de hipertensão arterial sistêmica, tiveram piores resultados. Em relação à escolaridade não houve variação quanto aos anos de estudo, predominando a saúde como regular.

As descobertas do estudo apontam possibilidades de intervenção para os profissionais da saúde, podendo facilitar o desenvolvimento de atividades que fomentem a melhora da saúde percebida pelos idosos com câncer, dentre as quais estão o estímulo ao apoio familiar e a manutenção de atividades laborais.

As limitações deste estudo estiveram relacionadas ao levantamento bibliográfico, restrito para estudos relacionados a autopercepção de saúde em associação com neoplasias. Espera-se que os resultados desta pesquisa possam contribuir para o desenvolvimento de próximos estudos acerca da temática, além de favorecer os profissionais da saúde com o conhecimento dos fatores que interferem na saúde autorreferida. Proporcionando reflexos em sua prática profissional para um cuidado integral, e que valorize a integridade e independência dos idosos para uma consequente melhora na sua qualidade de vida.

content/uploads/2012/06/Condicoes-Cronicas_flavio1.pdf.

4. Instituto Nacional de Câncer. Estimativa 2014: incidência de câncer no Brasil. Rio de Janeiro: Inca. 2013. [citado 2014 mar 16]. Disponível em:

http://www.inca.gov.br/estimativa/2014/estimativa24012014.pdf.

5. Cabrera MAS, Andrade SM, Wajngarten M. Causas de mortalidade em idosos: estudo de seguimento de nove anos. Geriatria \& Gerontologia. 2007;1(1):14-20. [citado 2014 mar 16]. Disponível em:

http://www.sbgg.org.br/profissionais/arquivo/revista/volume1numero1/completo.pdf.

6. Lima-Costa MF, Facchini LA, Matos DL, Macinko J. Mudanças em dez anos das desigualdades sociais em saúde dos idosos brasileiros (1998-2008). Rev Saúde Pública. 2012;46(supl):1007. 
7. Jylhä M. What is self-rated health and why does it predict mortality? Towards a unifi ed conceptual model. Soc Sci Med. 2009;69(3):307-16

8. Hartmann A. Fatores associados à autopercepção de saúde em idosos de Porto Alegre. [Tese]. Porto Alegre: Instituto de Geriatria e Gerontologia da Pontifícia Universidade Católica do Rio Grande do Sul; 2008. 73p.

9. Freitas DHM, Campos FCA, Linhares LQ, Santos CR, Ferreira $C B$, Diniz BS, Tavares A. Autopercepção da saúde e desempenho cognitivo em idosos residentes na comunidade. Rev Psiq Clín. 2010;37(1)32-5.

10. Söderhamn O. Phenomenological perspectives on self-care in aging. Clinical Interventions in Aging. 2013;8:605-8.

11. Silva T, Menezes P. Autopercepção de saúde: um estudo com idosos de baixa renda de São Paulo. Rev. Med. 2007;86(1):2838 , p. 30.

12. Jóia LC, Ruiz T, Donalísio MR. Grau de satisfação com a saúde entre idosos do município de Botucatu, Estado de São Paulo, Brasil. Epidemiol. Serv.Saúde. 2008;17(3):187-94. 13. Ji-Young A, Kyungeh U, O'connor L, Wexler S. Life Satisfaction, Self-esteem and assessment of health status among Elder Korean women: focus on living arrangements. J Transcult Enfermagem. 2008;19(2):151-60.

14. Pinto JM, Neri AL. Factors associated with low life satisfaction in community-dwelling elderly: FIBRA Study. Cadernos de Saúde Pública. 2013;29(12):2447-2458. [acesso 2014 mar 18].

Disponível em:

http://www.scielo.br/pdf/csp/v29n12/v29n12a10.pdf.

15. Kim SY, Sok SR. Relations between the state of health, family support and life satisfaction perceived older Korean adults. International journaul of nursing practice. $2012 ; 18(4): 325-31$.

16. Pagotto V, Nakatani A, Silveira E. Fatores associados à autoavaliação de saúde ruim em idosos usuários do Sistema Único de Saúde. Cad. Saúde Pública. 2011;27(8):1593-1602. 17. Santos M, Mattos I. Condições de vida e saúde da população idosa do Município de Guaramiranga-CE. Epidemiol. Serv. Saúde. 2011;20(2):193-201.

18. Santiago I. Condições associadas à saúde auto-referida em homens idosos de Juiz de Fora, MG. [dissertação]. Rio de Janeiro: Escola Nacional de Saúde Pública Sergio Arouca; 2009. $76 \mathrm{p}$.

19. Souza L, Lautert L, Hilleshein E. Trabalho voluntário, características demográficas, socioeconômicas e autopercepção da saúde de idosos de Porto Alegre. Rev Esc Enferm USP. 2010;44(3):561-9.

20. Sanchez K, Ferreira N, Andrade G, Costa D. Apoio social à família do paciente com câncer: identificando caminhos e direções. Rev Bras Enferm. 2010;63(2):299-9.

21. Rodrigues MVC, Ferreira ED, Menezes TMO. Comunicação da enfermeira com pacientes portadores De câncer fora de possibilidade de cura. Rev. Enferm. UERJ. 2010;18(1):86- 91. 22. Salci M, Marcon S. De cuidadora a cuidada: quando a mulher vivencia o câncer. Texto contexto-Enferm. 2008;17(3):544-51.

Artigo recebido em 06/12/2012.

Aprovado para publicação em 13/03/2014.

Artigo publicado em 30/09/2014. 